\title{
Can a once-daily oral formulation treat symptoms of uterine fibroids without causing hot flashes or bone loss?
}

$\overline{\text { FAST }}$

TRACK

Two 24-week phase 3 trials evaluated efficacy of placebo vs oral relugolix

$40 \mathrm{mg}$ with $1 \mathrm{mg}$ estradiol and

$0.5 \mathrm{mg}$ norethindrone acetate vs oral relugolix alone followed by the relugolix combination therapy

\section{Menstrual bleeding was significantly reduced} among women with uterine fibroids who took a new once-daily combination of relugolix (an oral gonadotropinreleasing hormone receptor antagonist) plus estrogen/ progestin versus placebo. There was also a significant reduction in uterine volume and pain as reported on a numerical rating scale, and bone mineral density was preserved. There was no increase in hot flashes (as is seen with relugolix when administered alone).

Al-Hendy A, Lukes AS, Poindexter AN 3rd, et al. Treatment of uterine fibroid symptoms with relugolix combination therapy. N Engl J Med. 2021;384:630-642. doi: 10.1056/ NEJMoa2008283

\section{EXPERT COMMENTARY}

Steven R. Goldstein, MD, NCMP, CCD, is Professor of Obstetrics and Gynecology, New York University Grossman School of Medicine, Directory of Gynecologic Ultrasound and Co-Director of Bone Densitometry, New York University Langone Medical Center, New York. Dr. Goldstein serves on the OBG MANAGEMENT Board of Editors.

y age 50 , approximately $70 \%$ of White women and $80 \%$ of Black women will have uterine fibroids. ${ }^{1}$ Of these, about $25 \%$ will have symptoms-most often including heavy menstrual bleeding, ${ }^{2}$ and associated pain the second most common symptom. ${ }^{3}$ First-line treatment has traditionally been hormonal contraceptives.

The author reports being an advisory board member for AbbVie and Myovant.

doi: 10.12788/obgm.0080
Injectable gonadotropin-releasing hormone (GnRH) antagonist like leuprolide acetate have been commonly employed, although their actual approved indication is "for concomitant use with iron therapy for preoperative hematologic improvement of patients with anemia caused by uterine leiomyomata (fibroids)." Recently, an oral GnRH antagonist, elagolix, combined with estrogen and progestogen, was approved for treatment of uterine fibroids for up to 24 months. However, it is dosed twice per day because of its short half-life and results in a loss of bone mineral density at 1 year. ${ }^{5,6}$

\section{Details of the studies}

Al-Hendy and colleagues report on two double-blind 24-week phase 3 trials involving women with heavy menstrual bleeding associated with fibroids. There were just under 400 women in each trial. There was a 1:1:1 randomization to: placebo, once-daily oral relugolix $40 \mathrm{mg}$ with $1 \mathrm{mg}$ estradiol and $0.5 \mathrm{mg}$ norethindrone acetate, or oral 
relugolix by itself for 12 weeks followed by the combination for 12 weeks (referred to as the "delayed relugolix combination therapy" arm).

Results. The primary end point was the percentage of patients who had a volume of menstrual blood loss less than $80 \mathrm{~mL}$ and a $\geq 50 \%$ reduction in blood loss volume as measured by the alkaline hematin method. The baseline blood loss in these studies ranged from approximately 210-250 mL. Secondary end points included amenorrhea, volume of menstrual blood loss, distress from bleeding and pelvic discomfort, anemia, pain, uterine volume, and the largest fibroid volume.

In trials one and two, $73 \%$ and $71 \%$ of patients in the relugolix combination groups, respectively, achieved the primary endpoint, compared with $19 \%$ and $15 \%$ in the placebo groups $(P<.001)$. In addition, all secondary endpoints except largest fibroid volume were significantly improved versus placebo. Adverse events, including any change in bone mineral density, were no different between the combination and placebo groups. The delayed combination groups did have more hot flashes and diminished bone density compared with both the placebo and combination groups.

\section{Strengths and weaknesses}

The studies appropriately enrolled women with a mean age of 41-42 years and a mean BMI $>30 \mathrm{~kg} / \mathrm{m}^{2}$, and more than $50 \%$ were African American. Thus, the samples are adequately representative of the type of population most likely to have fibroids and associated symptoms. The results showed the advantages of built-in "add back ther-

\section{References}

1. Wise LA, Laughlin-Tommaso SK. Epidemiology of uterine fibroids: from menarche to menopause. Clin Obstet Gynecol. 2016;59:2-24.

2. Borah BJ, Nicholson WK, Bradley L, et al. The impact of uterine leiomyomas: a national survey of affected women. Am J Obstet Gynecol. 2013;209:319.e1-319.e20.

3. David M, Pitz CM, Mihaylova A, et al. Myoma-associated pain frequency and intensity: a retrospective evaluation

\section{WHAT THIS EVIDENCE MEANS FOR PRACTICE}

Fibroids are extremely common in clinical practice, with their associated symptoms depending greatly on size and location. In many patients, symptoms are serious enough to be the most common indication for hysterectomy. In the past, combination oral contraceptives, injectable leuprolide acetate, and more recently, a $\mathrm{GnRH}$ antagonist given twice daily with estrogen/progestogen add-back have been utilized. The formulation described in Al-Hendy and colleagues' study, which is dosed once per day and appears to not increase vasomotor symptoms or diminish bone mass, may provide a very nice "tool" in the clinician's toolbox to either avoid any surgery in some patients (likely those aged closer to menopause) or optimize other patients preoperatively in terms of reversing anemia and reducing uterine volume, thus making any planned surgical procedure safer.

STEVEN R. GOLDSTEIN, MD, NCMP, CCD

apy" with estrogen plus progestogen, as the vasomotor symptoms and bone loss that treatment with a GnRH antagonist alone produces were reduced.

Although the trials were only conducted for 24 weeks, efficacy was seen as early as 4 weeks, and was clearly maintained throughout the full trials-and there is no scientific reason to assume it would not be maintained indefinitely. However, one cannot make a similar assumption about long-term safety. As another GnRH antagonist, with a shorter half-life requiring twice-daily-dosing with add back therapy, has been approved for use for 2 years, it is likely that the once-daily formulation of combination relugolix will be approved for this timeframe as well. Still, with patients' mean age of 41-42 years, what will clinicians do after 2-year treatment? Clearly, study of long-term safety would be valuable.

of 1548 myoma patients. Eur J Obstet Gynecol Reprod Biol. 2016;199:137-140.

4. Lupron Depot [package insert]. North Chicago, IL: AbbVie Inc.; 2018.

5. Schlaff WD, Ackerman RT, Al-Hendy A, et al. Elagolix for heavy menstrual bleeding in women with uterine fibroids. $N$ Engl J Med. 2020;382:328-340.

6. Oriahnn [package insert]. North Chicago, IL: AbbVie Inc.; 2020.
FAST

TRACK

In trials one and

two, $73 \%$ and

$71 \%$ of patients

in the relugolix

combination

groups, respectively,

achieved a volume

of menstrual blood

loss less than

$80 \mathrm{~mL}$ and a

$\geq 50 \%$ reduction in

blood loss volume 